\title{
HIGHLIGHTS
}

\section{Histone serotonylation boosts neuronal transcription}

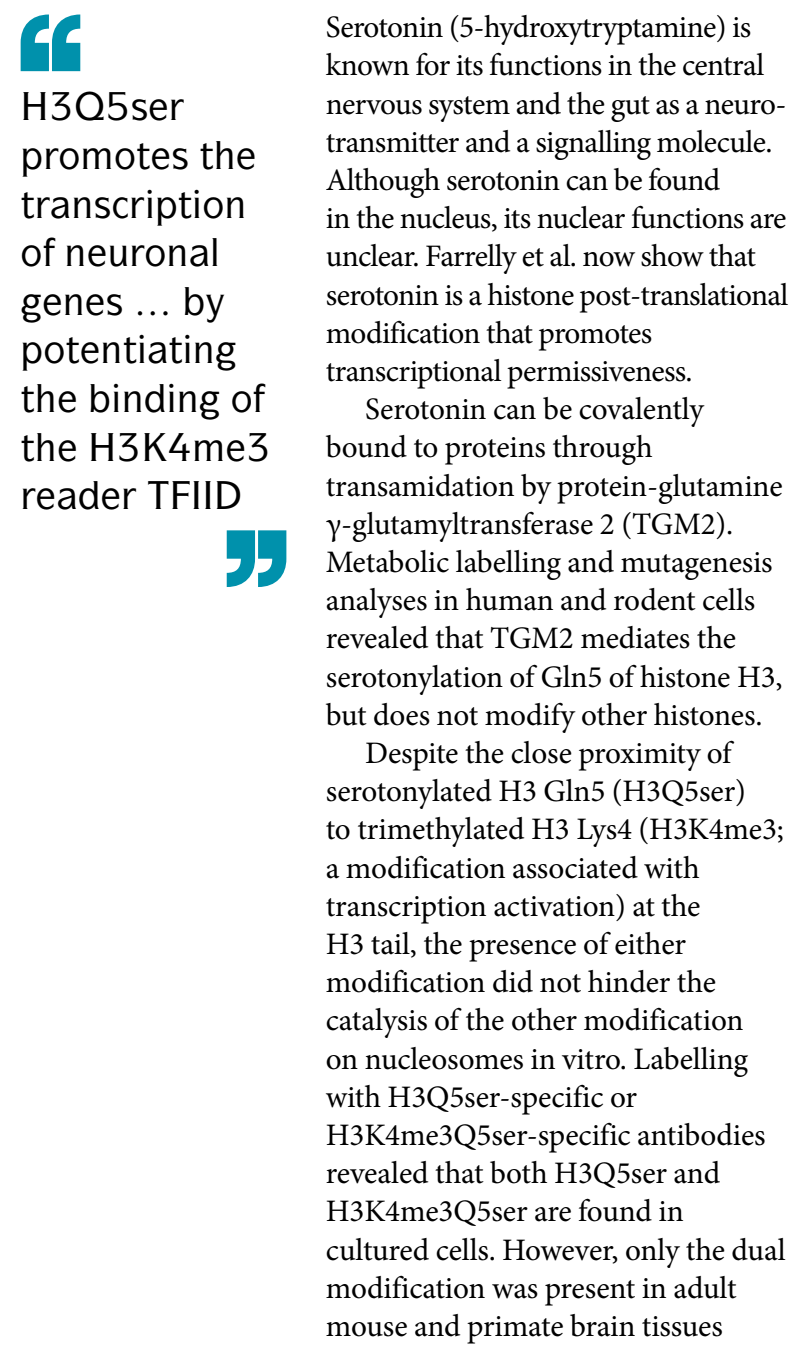

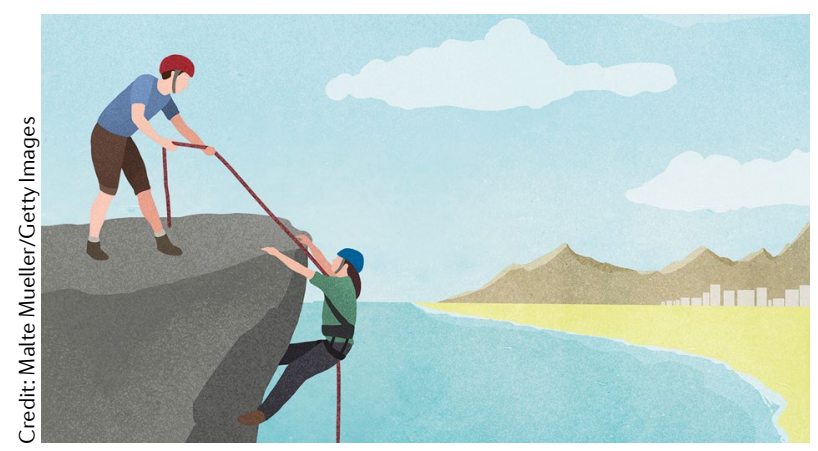

and in fruit fly larvae. Overall, the dual modification was ubiquitously present in various mammalian serotonin-producing tissues and, especially in the brain, also in non-serotonergic cell types.

To examine the role of H3K4me3Q5ser in cell differentiation, the levels of the dual modification were measured by chromatin immunoprecipitation and sequencing (ChIP-seq) in human pluripotent stem cell-derived serotonergic neurons, before and after differentiation. Differentiation led to a marked increase in $\mathrm{H} 3 \mathrm{~K} 4 \mathrm{me} 3 \mathrm{Q} 5$ ser levels, especially at promoters of activated neuronal genes. This finding was corroborated in mouse embryonic brains examined at a time period in which serotonergic neurons become fully differentiated.

The role of $\mathrm{H} 3 \mathrm{Q} 5$ ser was further examined in the in vitro differentiation model of rat RN46A-B14 serotonergic cells. In differentiating cells, ChIP-seq signals of the antibody targeting the dual modification markedly increased at many gene promoters, without concomitant increase in signals of the antibody targeting $\mathrm{H} 3 \mathrm{~K} 4 \mathrm{me} 3$ alone. This indicated that during differentiation, $\mathrm{H} 3 \mathrm{Q} 5$ ser levels increased without concomitant increase in H3K4me3 levels. Increased H3K4me3Q5ser again correlated with gene activation. Interestingly, H3K4me3Q5ser enrichment occurred at about two-thirds of genes that, in undifferentiated cells, had high levels of both $\mathrm{H} 3 \mathrm{~K} 4 \mathrm{me} 3$ and the repressive modification $\mathrm{H} 3 \mathrm{~K} 27 \mathrm{me} 3$ ('bivalent genes'), indicating that $\mathrm{H} 3 \mathrm{Q} 5$ ser may act to circumvent the repression of bivalent genes during differentiation.

TGM2 inhibition during RN46A-B14 cell differentiation decreased the expression of the H3K4me3Q5ser-regulated genes. Similarly, expressing a histone $\mathrm{H} 3$ mutant that cannot be serotonylated altered the expression patterns of many H3K4me3Q5ser-regulated genes and considerably reduced neurite length, suggesting that H3Q5ser is required for the differentiation of RN46A-B14 cells.

To investigate the effect of $\mathrm{H} 3 \mathrm{Q} 5 \mathrm{ser}$ on protein interactions of the $\mathrm{H} 3$ tail, synthetically modified $\mathrm{H} 3$-tail peptides were immunoprecipitated to identify their binding partners. The binding of dozens of known H3K4me3-binding proteins ( $\mathrm{H} 3 \mathrm{~K} 4 \mathrm{me} 3$ readers) was altered by the presence of Q5ser in the peptide. Among the H3K4me3 readers that exhibited Q5ser-enhanced binding were 15 members of the transcription factor IID (TFIID) complex, which functions in transcription initiation. ChIP-seq of a TFIID component in RN46A-B14 cells before and after differentiation revealed enrichment of $\mathrm{H} 3 \mathrm{~K} 4 \mathrm{me}$ 3Q5ser compared with $\mathrm{H} 3 \mathrm{~K} 4 \mathrm{me} 3$ in differentiated cells, suggesting that serotonylation of $\mathrm{H} 3 \mathrm{Q} 5$ potentiates transcription initiation through TFIID during differentiation.

Serotonylation of H3Q5 is the first endogenous monoaminyl modification, and the first non-methyl post-translational modification of Gln, to be identified in histones. H3Q5ser promotes the transcription of neuronal genes during neuronal cell differentiation by potentiating the binding of the $\mathrm{H} 3 \mathrm{~K} 4 \mathrm{me} 3$ reader TFIID at the gene promoters. Future studies will focus on identifying H3Q5ser readers and erasers and on other functions of $\mathrm{H} 3 \mathrm{Q} 5 \mathrm{ser}$, for example, in the gut, and its possible contribution to pathophysiological conditions in the brain associated with aberrant serotonin function, such as mood-related disorders.

Eytan Zlotorynski

ORIGINAL ARTICLE Farrelly, L. A. et al. Histone serotonylation is a permissive modification that enhances TFIID binding to H3K4me3. Nature 567 . 535-539 (2019) 